\title{
Atmospheric Parameters and Luminosities of Nearby M Dwarfs - Estimating Habitable Exoplanet Detectability with the E-ELT
}

\author{
Gustavo F. Porto de Mello†, Riano E. Giribaldi, \\ Diego Lorenzo-Oliveira ${ }^{1}$ and Nathália M. Paes Leme
}

Observatório do Valongo, Universidade Federal do Rio de Janeiro, Ladeira do Pedro Antonio 43, Rio de Janeiro, RJ, CEP: 20080-090, Brazil

email: gustavo@astro.ufrj.br

\begin{abstract}
We derive $T_{\text {eff }}$ and $[\mathrm{Fe} / \mathrm{H}]$ for a sample of 72 nearby $\mathrm{M}$-dwarfs with Hipparcos parallaxes and $\delta<+30$. Spectra, acquired at the Observatório do Pico dos Dias, Brazil, have $\mathrm{R}$ $=10,000$ and $\mathrm{S} / \mathrm{N} \gtrsim 100$ for nearly all targets in the $\lambda \lambda 8380-8880$ range. Atmospheric parameters were derived from VJHK colors and a system of spectral line indices calibrated against sample stars with interferometric $T_{\text {eff }}$ and $[\mathrm{Fe} / \mathrm{H}]$ from detailed analysis of FGK binary companions. A PCA method of calibration yields internal errors within $70 \mathrm{~K}$ and 0.1 dex for $T_{\text {eff }}$ and $[\mathrm{Fe} / \mathrm{H}]$. For 18 stars we present the first $T_{\text {eff }}$ or $[\mathrm{Fe} / \mathrm{H}]$ derivation in the literature. We compute the star's luminosities, calculate the position of their habitable zones and estimate that, were all of they to harbour rocky planets inside their HZ, 15-20 of these would be detectable by the E-ELT Planetary Camera and Spectrograph.
\end{abstract}

Keywords stars: abundances - stars: late-type - stars: low-mass - (stars:) planetary systems

\section{Introduction}

Low mass M dwarf stars occupy the center stage in modern searches for habitable planets in the solar neighborhood owing to: 1) their very large number densities, accounting for more than $70 \%$ of nearby stars, making them the most likely hosts of habitable planets (Henry et al. 1994); 2) the relative ease with which rocky planets may be discovered orbiting them, and the high occurrence rate of such planets (Cassan et al. 2012) at 1 planet per low mass star with period $<50$ days; 3 ) the tantalizing observability (Turbet et al. 2016) of such planets in the future $\sim 40 \mathrm{~m}$-class telescopes, following the discovery (Anglada-Escudé et al. 2016) of an Earth-like, possibly habitable (Ribas et al. 2016) planet in the late-type M dwarf Proxima Centauri, the closest stellar system to the Sun. Exoplanetology demands the systematic and accurate knowledge of the atmospheric parameters, effective temperature $T_{\text {eff }}$ and metallicity $[\mathrm{Fe} / \mathrm{H}]$, of nearby $\mathrm{M}$ dwarfs. This knowledge remains wanting in the face of the high importance of these systems, yet there has been progress in their distance determinations and census completeness for the nearby population. Ongoing surveys keep enlarging the samples of probable nearby unstudied M dwarfs (Winters et al. 2015). We aim to take advantage of these new candidate lists to provide intermediate resolution spectroscopic data for nearby, southern $\mathrm{M}$ dwarfs, contributing to the systematic determination of their $T_{\text {eff }}$ and $[\mathrm{Fe} / \mathrm{H}]$. Finally, the discovery of an Earthlike and possibly habitable planet in Proxima Centauri highlights the possibility that other nearby $\mathrm{M}$ dwarfs harbor equally interesting, potentially directly

$\dagger$ Based on observations collected at the Observatório do Pico dos Dias (OPD), operated by the Laboratório Nacional de Astrofísica, CNPq, Brazil. 


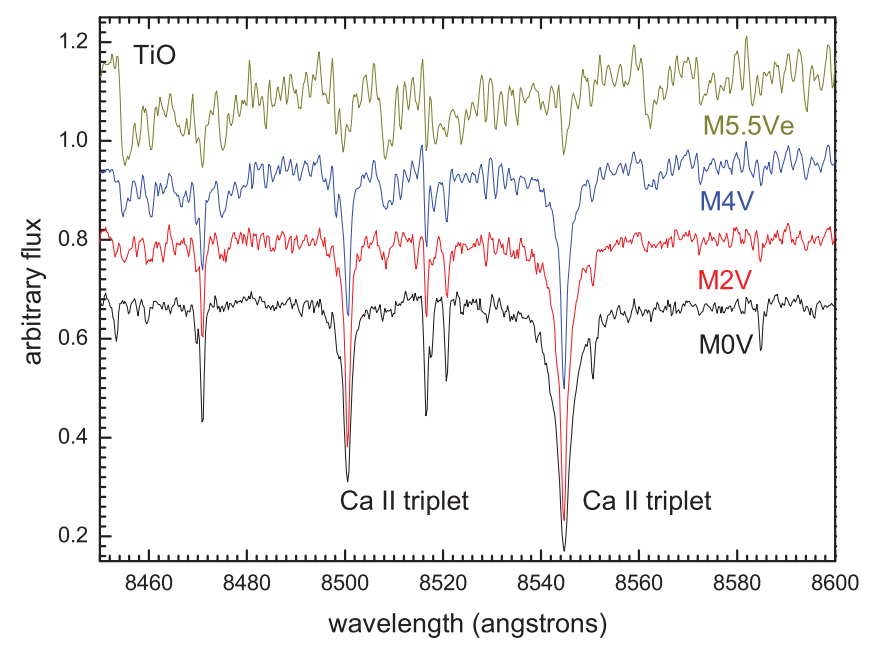

Figure 1. Sample spectra: the TiO $\lambda 8455$ bandhead and two of the Ca II triplet lines are indicated.

observable planets. We use our new $T_{\text {eff }}$ and $[\mathrm{Fe} / \mathrm{H}]$ data to obtain luminosities and reassess the dimensions of the habitable zones of nearby $\mathrm{M}$ dwarfs, estimating the possible fraction of their potentially habitable rocky planets that might be directly observable by the next generation of $>30 \mathrm{~m}$ telescopes.

\section{Observations}

Spectra were obtained at the coudé spectrograph of the $1.60 \mathrm{~m}$ telescope of the Observatório do Pico dos Dias (OPD), operated by the Laboratório Nacional de Astrofíísica (Brazil), and were mostly exposed to $\mathrm{S} / \mathrm{N}>100$ (Fig. 1) at a spectral resolution of $0.85 \AA(\mathrm{R}=10,000)$, the median of the $\mathrm{S} / \mathrm{N}$ values being $\sim 150$ and reaching out to $\sim 250$ in the best cases. Coverage is $\lambda \lambda 8380-8880$. A total of 72 stars had spectra of sufficient quality, corresponding to $81 \%$ of the population of these objects within 10 parsecs, with Hipparcos parallaxes and below $\delta<+30$.

\section{Determination of atmospheric parameters}

We used VJHK colors and a system of 68 initial spectral line indexes to calibrate $T_{\text {eff }}$ and $[\mathrm{Fe} / \mathrm{H}]$ by means of a PCA analysis. For the $T_{\text {eff }}$ calibration we demanded the correlation coefficient of the index versus $T_{\text {eff }}$ regression to be $\mathrm{R} \geqslant 0.80$ and kept 38 indices; for $[\mathrm{Fe} / \mathrm{H}]$ we enforced $\mathrm{R} \geqslant 0.70$ and kept 6 indices only. For the $T_{\text {eff }}$ calibration 7 PCA components explained $90 \%$ of the variance, while 3 PCA components achieved this for $[\mathrm{Fe} / \mathrm{H}]$. The final calibrations employed only the two most significant PCA components for each atmospheric parameter. Internal uncertainties respectively for $T_{\text {eff }}$ and $[\mathrm{Fe} / \mathrm{H}]$ are $70 \mathrm{~K}$ and $0.11 \mathrm{dex}$. The $T_{\text {eff }}$ values for the calibrating stars come mostly from interferometric, model-free determinations taken from Maldonado et al. (2015) and Rojas-Ayala et al. $(2012)$; the $[\mathrm{Fe} / \mathrm{H}]$ values come exclusively from binary systems for which $[\mathrm{Fe} / \mathrm{H}]$ is known from a spectroscopic model atmosphere analysis of the primary FGK-star (Neves et al. 2014). For 18 stars no published either $T_{\text {eff }}$ or $[\mathrm{Fe} / \mathrm{H}]$ was found in the literature, our results being thus the first ever atmospheric parameters provided for these objects. 


\section{Exoplanet Detectability}

The upcoming 39m E-ELT telescope is designed, e.g., with the Planetary Camera and Spectrograph (PCS), to obtain a star-planet contrast in the $10^{-7}$ to $10^{-8}$ range at $1 \mu \mathrm{m}$. An inner working angle of $\sim 7 \lambda / \mathrm{D}$ corresponds to 38 mas at one parsec (Turbet et al. 2016), which is the angular separation of the Proxima Centauri planet for the equivalent irradiance the Earth receives from the Sun, $S_{\text {eff }} \sim 1$. Under the hypothesis that $\sim 5 \lambda / D$ achieves direct imaging detection for a rocky, high albedo planet with one Earth-radius (Turbet et al. 2016), a working limit of $\sim 30$ mas is inferred. The angular separation, in mas, between a potentially habitable putative planet and its host star is given by the star's luminosity in solar units $\mathrm{L} / \mathrm{L}_{\odot}$ and its distance $\mathrm{d}$ in parsecs by $\alpha_{\text {plan }}=960$ $\left[\left(\mathrm{L} / \mathrm{L}_{\odot}\right) / \mathrm{d}\right]^{-1 / 2}$. We calculated luminosities for the $\mathrm{M}$ dwarf stars in our sample using our derived $T_{\text {eff }}$ and $[\mathrm{Fe} / \mathrm{H}]$, Hipparcos parallaxes and up-to-date (Mann et al. 2015) bolometric corrections. Observability is also set by the star's apparent magnitude and distance of the star-planet system, but under optimistic conditions the above formula allows the estimation of the detectability of hypothetical rocky planets sited inside the habitable zone (HZ) of our sample's stars by means of the PCS-E-ELT. For $\mathrm{S}_{\text {eff }} \sim 1$ there could be 15 stars in our sample for which a rocky planet would be detectable inside the HZ. Assuming the rocky planets are at the very limit of the theoretical HZ - which is $\mathrm{S}_{\text {eff }}$ $\sim 0.3$ (the so called maximum greenhouse limit) (Kasting et al. 1993) - would improve detectability due to a larger star-planet angular separation, bringing a total of 22 possible systems of our sample under the direct imaging capabilities of the PCS-E-ELT.

\section{Perspectives}

Our method can be much improved by a more detailed definition of the spectral indices, isolating the sensitivity of specific groups of transitions to $T_{\text {eff }}$ and $[\mathrm{Fe} / \mathrm{H}]$ and possibly even to surface gravity log g (which we did not determine here). We also plan to extend the calibration to include more colors, such as griz, (V-R) and (V-I) colors (Winters et al. 2015) as well as WISE and Gaia colors (Mann et al. 2015). The inclusion of a Bayesian approach with a large quantity of colors and indices has been shown to improve considerably the $T_{\text {eff }}$ and $[\mathrm{Fe} / \mathrm{H}]$ determinations (Lorenzo-Oliveira 2016). More observations are planned at OPD: the instrumentation has been improved, with a new CCD and better efficiency at the coudé spectrograph, with which it is possible to extend the sample at least to $\mathrm{V} \sim 12$. Potentially all southern $\mathrm{M}$ dwarfs down to type $\sim \mathrm{M} 3 \mathrm{~V}-\mathrm{M} 4 \mathrm{~V}$, and within 10 parsecs, are accessible to the OPD spectrograph for reasonable integration times.

\section{References}

Anglada-Escudé, G., Amado, P. J., Barnes J. et al. 2016, Nature, 536, 437

Cassan, A., Kubas, D., Beaulieu, J.-P., et al. 2012, Nature, 481, 167

Henry, T. J., Kirkpatrick, J. D., \& Simmons, D. A. 1994, AJ, 108, 1437

Kasting, J. F., Whitmire, D. P., \& Reynolds, R. T. 1993, Icarus, 101, 108

Lorenzo-Oliveira, D. L. 2016, PhD thesis, Observatório do Valongo, Universidade Federal do Rio de Janeiro

Maldonado, J., Affer, L., Micela, G. et al. 2015, A\&A, 577, A312

Mann, A. W., Feiden, G. A., Gaidos, E. et al. 2015, ApJ, 804, 64

Neves, V., Bonfils, X., Santos, N. C. et al. 2014, A\& A, 568, A121

Ribas, I, Bolmont, E., Selsis F. et al. 2016, arXiv:1608:06813,

Rojas-Ayala, B., Covey, K. R., Muirhead P. S. et al. 2012, ApJ, 748, 93

Turbet, M., Leconte, J., Selsis F. et al. 2016, arXiv:1608:06827,

Winters, J. G., Henry, T. J., Lurie, J. C. et al. 2015, AJ, 149, 5 